EPJ Web of Conferences 114, 02030 (2016)

DOI: $10.1051 /$ epjconf/201611402030

(c) Owned by the authors, published by EDP Sciences, 2016

\title{
Unsteady flow over flexible wings at different low Reynolds numbers
}

\author{
Mustafa Serdar Genç ${ }^{1}$, Mustafa Özden ${ }^{1}$, Halil Hakan Açikel ${ }^{1, a}$, Hacımurat Demir ${ }^{1,2}$, lliasbek Isabekov ${ }^{1}$ \\ ${ }^{1}$ Wind Engineering and Aerodynamic Research Group, Department of Energy Systems Engineering, Erciyes University, Kayseri, Turkey \\ ${ }^{2}$ Department of Mechanical Engineering, Aksaray University, Aksaray, Turkey
}

\begin{abstract}
In this study, unsteady flow around flexible membrane wing which had aspect ratio of 1 (AR=1) was investigated experimentally at various Reynolds numbers $(\mathrm{Re}=25000$ and $\mathrm{Re}=50000)$. Smoke-wire technique for flow visualization over the flexible membrane wing was utilized in the experiments. Digital Image Correlation system (DIC) was used for measuring deformation of $\mathrm{AR}=1$ flexible membrane wing. Instantaneous deformation measurements of membrane wing were combined with the flow field measurements. In low aspect ratio flexible membrane wings, unsteadiness includes tip vortices and vortex shedding, and the combination of tip vortices. In these types of wings, complex unsteady deformations occurred due to vortex shedding. The results showed that the increasing angle of attack results in increase of membrane deformation. Moreover, it was concluded that analysis of the instantaneous deformation revealed chordwise and spanwise, modes which were due to the shedding of leading-edge vortices as well as tip vortices. Consequently, vibrational mode decreased and maximum standard deviation location approached to the trailing edge by reason of increasing angle of attack.
\end{abstract}

\section{Introduction}

Recently, due to the advances in unmanned aerial vehicles (UAV), micro air vehicles (MAV) and wind turbines, aerodynamics researches concentrated on low Reynolds number aerodynamics, transition and laminar separation bubble (LSB) and its effects on aerodynamic performance [1-5]. In low Reynolds number aerodynamics, unsteadiness represents major problems. Furthermore, laminar separation bubble may cause negative effects, such as decreasing on lift, increasing on drag, reducing stability of the aircraft, noise and vibration in low Reynolds number aerodynamics. Membrane wings are used in many engineering applications such as microlight, yacht sails, parachutes, hang glider wings, paraglider and wings of small unmanned air vehicles go by the name of micro air vehicles (MAVs) [6]. Some studies show that flexible membrane wings may remarkably improve longitudinal static stability, delay stall and ensure a more favourable lift-to-drag ratio, and so enhance the overall aerodynamic performance when comparing with a rigid wing of similar geometry due to its aeroelastic effects and the adaptive inflation of the membrane skin.

Genç [7] investigated unsteady aerodynamics and flow-induced vibrations of a low aspect ratio rectangular membrane wing with excess length. The experiments for the membrane and rigid wing models are conducted in a closed-loop open-jet wind tunnel with a circular working section of $760 \mathrm{~mm}$ in diameter at angles of attack ranging from $0^{\circ}$ to $25^{\circ}$ at Reynolds number of 48,700 . Digital Image Correlation system is used for time-accurate measurements of membrane deformation. Furthermore, measurement of velocity field with Digital Particle Image Velocimetry system was conducted whereas normal forces of the wing were measured by means of a load-cell system. Obtained experimental results demonstrated that the camber of membrane wing with excess length excited the separated shear layer. This situation leads to increase the lift coefficient relatively more as compared to membrane wings without excess length. The membrane caused to generate a larger magnitude of the maximum camber in the both $2 \mathrm{D}$ and $3 \mathrm{D}$ rectangular membrane wings with excess length. Dominant vibrational modes were obtained as a spanwise mode of two at the angles of attack of $6-10^{\circ}$ and $19-22^{\circ}$. On the other hand, dominant spanwise modes were three at $11-18^{\circ}$ and $23-25^{\circ}$.

Rojratsirikul et al. [8] investigated two dimensional flexible membrane airfoils experimentally with respect to the effects of membrane pre-strain and excess length on unsteady fluid structure interactions of membrane airfoils The largest camber and strain were observed for the airfoil with excess length. They noticed that the onset of the membrane airfoil vibrations was postponed to a higher angle of attack for the airfoils with excess length. Additionally, both the mode number and Strouhal number were in tendency to decrease with increasing angle of attack. Rojratsirikul et al. [9] performed an experimental study of a low aspect ratio rectangular wing for a Reynolds number range of $2.4 \times 10^{4}-4.8 \times 10^{4}$. Time

\footnotetext{
a Corresponding author: hhacikel@erciyes.edu.tr
} 
accurate measurements of membrane deformation were added with the flow field measurements. Analysis of the fluctuating deformation detected chordwise and spanwise modes, which were due to the shedding of leading-edge vortices as well as tip vortices. It was seen that the second mode in the chordwise direction became dominant as the vortex shedding took place at higher angles of attack. The dominant frequencies of the membrane vibrations were similar to those of two-dimensional membrane airfoils. Additionally, it was seen that vortex shedding frequency from rigid wings showed significantly small effect of aspect ratio even when it was as low as unity.

Béguin and Breitsamter [10] studied on effects of membrane pre-stress on the aerodynamic characteristics of elasto-flexible morphing wing for applications of unmanned air vehicle. The results of force and deformation measurements conducted at several dynamic pressures explained that varying the pre-stress effectively permitted to influence the passive deformation of the wing surface and regulated the wing camber within a certain range at a given flow condition. In addition to this, it could be concluded that the characteristics of lift and drag were affected such that an improvement of the maximum lift-to-drag ratio between $6 \%$ and $23 \%$ of the lowest values at a given flow condition could be achieved.

Arbo's-Torrent et al. [11] studied on leading- and trailing-edge effects on the aeromechanics of membrane aerofoils which was made of black latex rubber sheet. Two different cross-sectional geometries in two different sizes were used to in order to explore the effects of leading-edge (LE) and trailing-edge (TE) support designs on the performance of membrane aerofoils. They noticed that the mean camber as well as membrane vibrations changed with size and geometry of the LE and TE supports. Furthermore, the LE/TE supports with a rectangular cross-section continuously ensured higher lift forces and higher mean camber deformations comparing the support with circular cross-section. In addition to this, it was seen that membrane vibrations were higher for aerofoils with LE/TE supports with rectangular crosssection. LE/TE supports deviated under aerodynamic loading and thereby changed the performance of the aerofoil. Albertani et al. [12] investigated the aerodynamic coefficients and elastic deformations of low aspect ratio flexible wings. They conducted the experiments in a low-speed wind tunnel utilizing a visual image correlation system and a six-component strain gauge sting balance. It was seen that both lift and static longitudinal stability were increased and hence remarkable advantages in a flexible membrane wing performance were obtained.

Lian et al. [13] reviewed the aerodynamics of membrane and corresponding rigid wings under the micro air vehicle flight conditions. They noticed that the membrane wing both delayed stall as well as adapted to the unsteady flight environments, and that the proper orthogonal decomposition method was an economic tool in order to identify the flow structure around a wing and in order to facilitate flow and vehicle control. Aono et al. [14] investigated the effects of spanwise flexibility on flapping wing aerodynamics computationally. They concluded that the spanwise wing deformation and the increased effective angle of attack of the deformed wing increased thrust generation. On the other hand, it was noticed that when the flexibility was too high, the wing tip and root moved in inconsistent directions, which caused deteriorated aerodynamic performance. Kuo and Hsieh [15] studied on unsteady flow structure and vorticity convection over NACA0012 airfoil oscillating at high reduced frequency. They noticed that the leading edge separation vortices were combined one by one into a vortex near the trailing edge and then shed together downstream into the wake. The wavelength of unsteady vortex structure over the airfoil was found to be remarkably different from that at low reduced frequencies. Furthermore, they also noted that the effect of different pivoting axis was insignificant on the unsteady flow structure at such high reduced frequency oscillations. Ringuette et al. [16] investigated the role of the tip vortex in the force generation of low-aspect-ratio normal flat plates experimentally. They noticed that tip vortex resulted in a significant maximum in the plate force. Furthermore, decrease in aspect ratio caused to increasing of plate drag coefficient.

\section{Experimental Set-up}

The experiments were performed in a low-speed, suctiontype wind tunnel (square working section of $500 \mathrm{~mm} \mathrm{x}$ $500 \mathrm{~mm}$ ) located at the Department of Energy Systems Engineering, University of Erciyes as shown in Figure 1. Turbulence intensity of the tunnel is $0.35 \%$ and the operating range of wind tunnel speed was 1-45 m/s. Flexible membrane wing and cross sectional area of the frames of the wing was shown in Figure 2. The wing had $125 \mathrm{~mm}$ chord length and $125 \mathrm{~mm}$ span length ( $\mathrm{AR}=1)$, was made from a black latex rubber sheet with a thickness of $\mathrm{t}=0.2 \mathrm{~mm}$, Young's modulus of $\mathrm{E}=2.2$ $\mathrm{MPa}$, and density of $\rho_{\mathrm{m}}=1 \mathrm{~g} / \mathrm{cm}^{3}$ was used. Experimental setup of DIC system for deformation measurement was given in Figure 3.

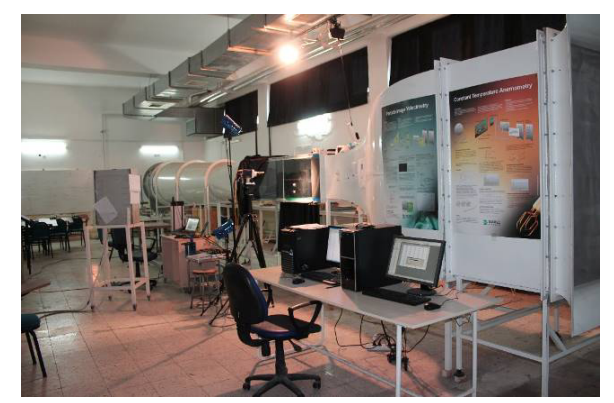

Figure 1. Photograph of the experimental set-up. 


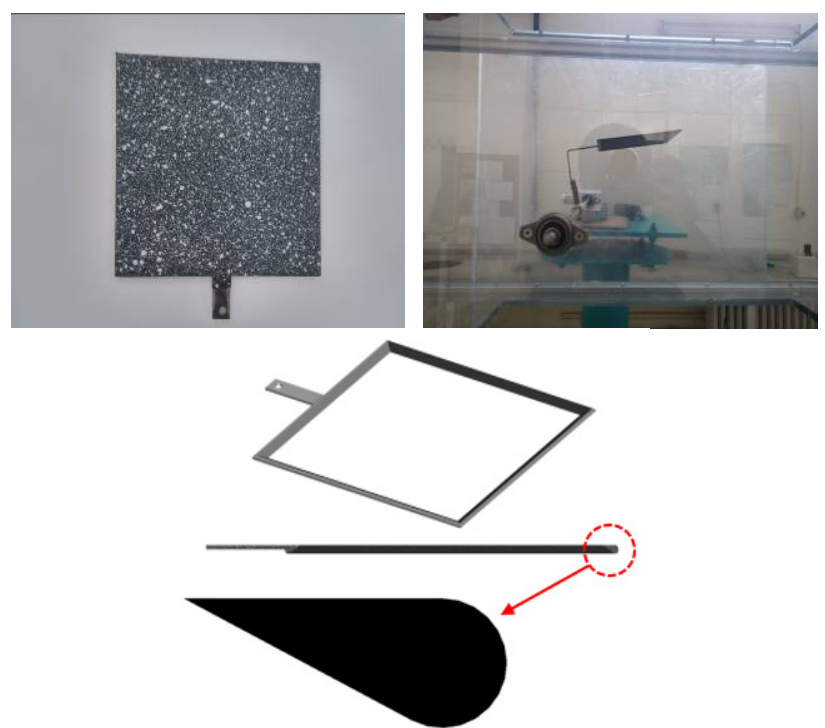

Figure 2. Schematic of the flexible membrane wing $(A R=1)$ with cross-section of the rigid frame in wind tunnel.

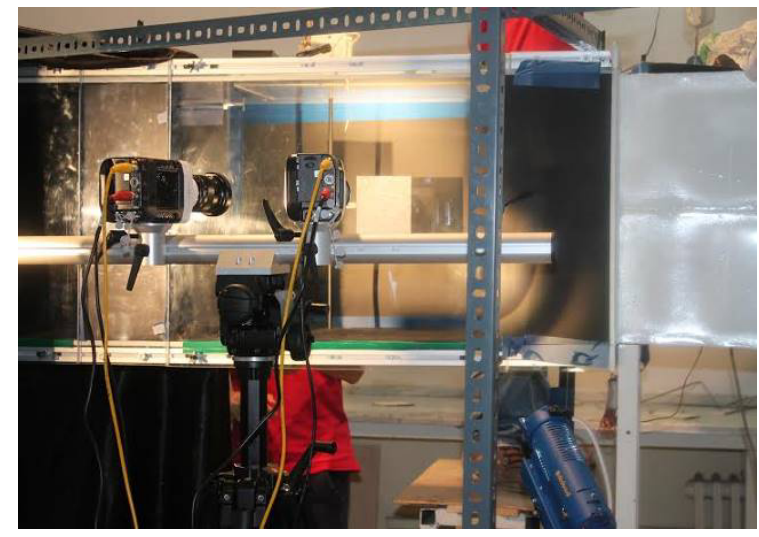

Figure 3. Photograph of the experimental set-up for DIC measurement.

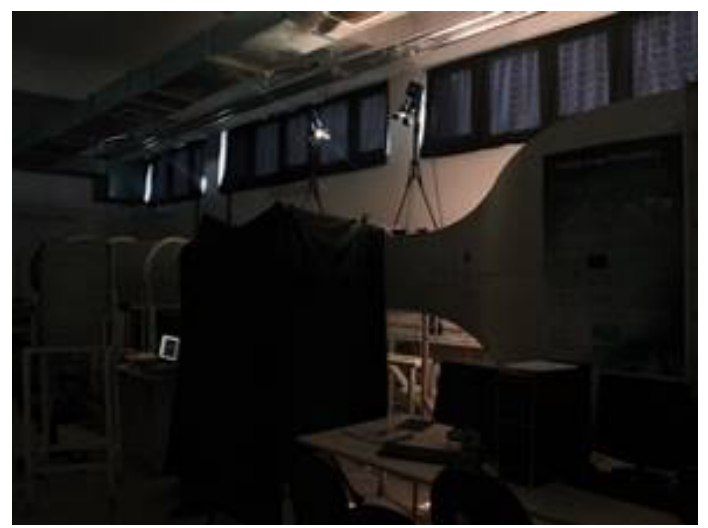

Figure 4. Dark medium for smoke-wire measurement

\subsection{Smoke-wire flow visualization and} deformation measurement

\subsubsection{Smoke-wire flow visualization}

The smoke-wire visualization method was employed for flow visualization in order to mark the streamlines. The smoke experiments were conducted on by a heated wire and oil supply system which sent oil from top of the wire and burning oil moved with the free stream. Dark medium for smoke-wire measurement is seen in Figure 4.

\subsubsection{Deformation measurement}

Digital Image Correlation (DIC) system was used to measure the membrane displacements. Full-field measurements were obtained by means of using the DANTEC Dynamics 3D High-Speed Image Correlation System (Q-450: the number of pixels was $1280 \times 800$ ). The frame rate was $1 \mathrm{kHz}$. DANTEC Al-11-BMB_9x9 calibration target was utilized. During the deformation measurement, 1000 frame was captured each experiment.

\section{Comparisons between Experimental and Numerical Results}

\subsection{Results of smoke-wire visualization}

Smoke-wire visualization results for $\mathrm{Re}=25000$ and $\mathrm{Re}$ $=50000$ are shown in Figure 5 and Figure 6. Firstly, smoke-wire experiment was carried out at constant $\mathrm{Re}=$ 25000 with angles of attack of $\alpha=12^{\circ}, 16^{\circ}, 20^{\circ}$ and $24^{\circ}$, respectively. As seen from the Figure 5, the formations of tip vortices were increased with increase in angle of attack from $\alpha=12^{\circ}$ to $\alpha=24^{\circ}$.

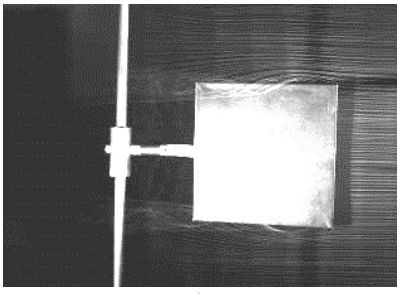

a)

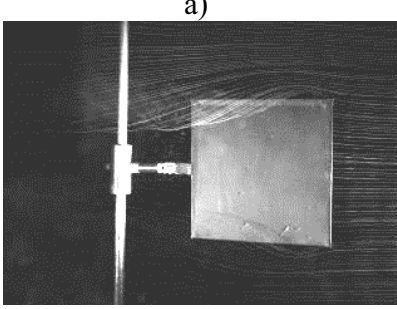

c)

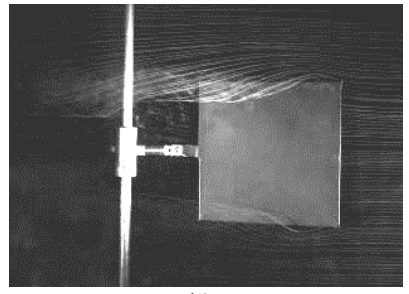

b)

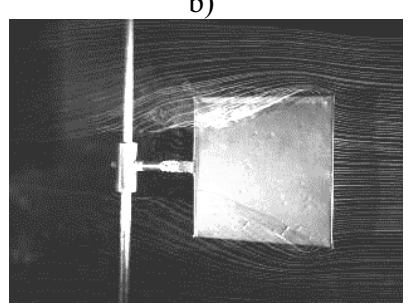

d)
Figure 5. Smoke-wire visualization results at $\operatorname{Re}=25000$ a) $\alpha=$ $12^{\circ}$, b) $\alpha=16^{\circ}$, c) $\alpha=20^{\circ}$ and d) $\alpha=24^{\circ}$

Additionally, tip vortices grew up from leading edge to the trailing edge of membrane wing. Then, smoke-wire experiment was carried out at $\mathrm{Re}=50000$ for the same angles of attack. Results of experiments were given in Figure 6. It is seen from the Figure 6 that increasing angle of attack results in increase tip vortex. Due to this, tip vortices affected the unsteady aerodynamics of the membrane wing. 


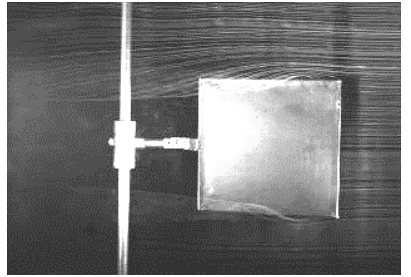

a)

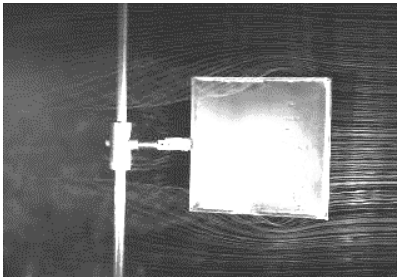

c)

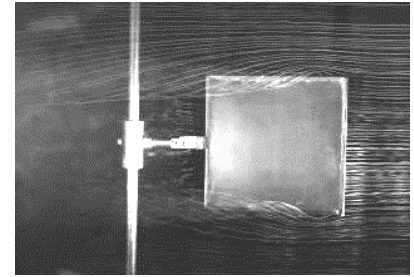

b)

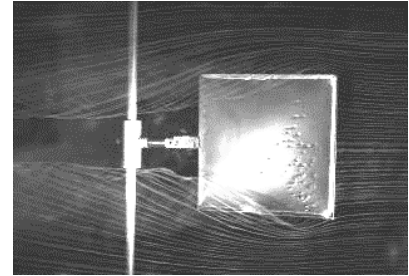

d)
Figure 6. Smoke-wire visualization results at $\operatorname{Re}=50000$ a) $\alpha=$ $12^{\circ}$, b) $\alpha=16^{\circ}$, c) $\alpha=20^{\circ}$ and d) $\alpha=24^{\circ}$

\subsection{DIC results}

Due to the unsteady flow over the low aspect ratio (AR $=$ 1) flexible membrane wing, deformations were observed over the related wing. Unsteady condition of membrane wing was investigated for $\mathrm{Re}=25000$ and $\mathrm{Re}=50000$ with different time intervals. Instantaneous displacement results for the flexible membrane wing at $\mathrm{Re}=25000$ and $\mathrm{Re}=50000$ with various angles of attack were given from Figure 7 to Figure 12.

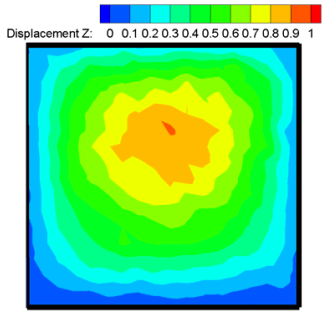

a) $\mathrm{t}=0.001 \mathrm{~s}$

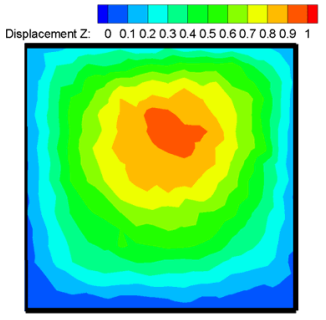

b) $\mathrm{t}=0.5 \mathrm{~s}$

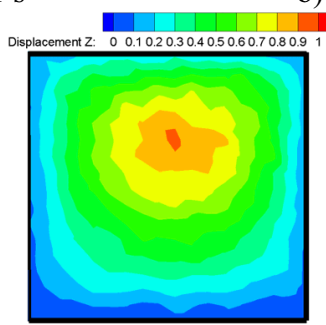

c) $\mathrm{t}=1 \mathrm{~s}$

Figure 7. Instantaneous deformation results for flexible membrane wing for $\mathrm{Re}=25000$ and $\alpha=16^{\circ}$

Deformation results for $\mathrm{Re}=25000$ with angles of attack $\alpha=16^{\circ}, \alpha=20^{\circ}$ and $\alpha=24^{\circ}$ were demonstrated in Figure 7 to Figure 9. It was understood from obtained results that increase angle of attack caused to increase in maximum instantaneous deformation. At same $\mathrm{Re}$ number and angle of attack condition (i.e. Figure 7a), location of the maximum instantaneous deformation contour varied due to unsteady flow condition.

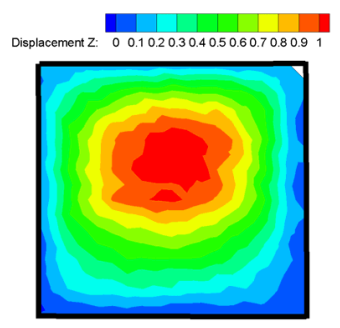

a) $\mathrm{t}=0.001 \mathrm{~s}$

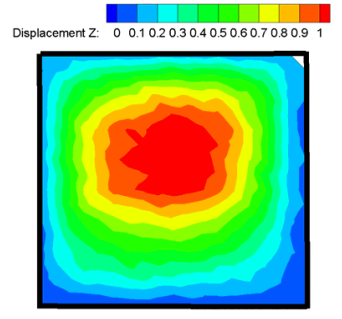

b) $\mathrm{t}=0.5 \mathrm{~s}$

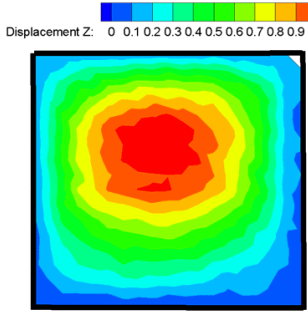

c) $\mathrm{t}=1 \mathrm{~s}$

Figure 8. Instantaneous deformation results for flexible membrane wing for $\mathrm{Re}=25000$ and $\alpha=20^{\circ}$

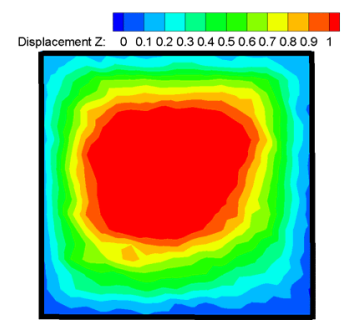

a) $\mathrm{t}=0.001 \mathrm{~s}$

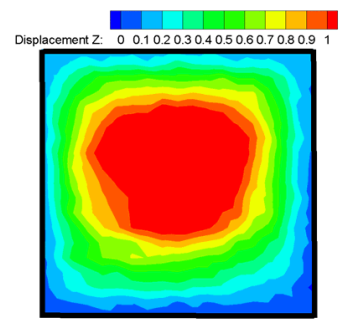

b) $\mathrm{t}=0.5 \mathrm{~s}$

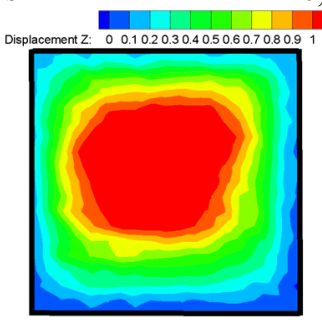

c) $\mathrm{t}=1 \mathrm{~s}$

Figure 9. Instantaneous deformation results for flexible membrane wing for $\mathrm{Re}=25000$ and $\alpha=24^{\circ}$

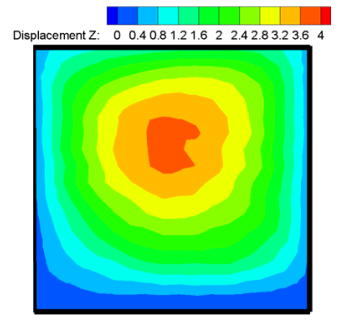

a) $\mathrm{t}=0.001 \mathrm{~s}$

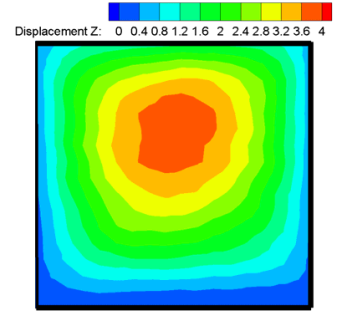

b) $t=0.5 \mathrm{~s}$

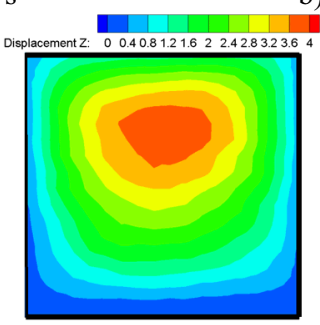

c) $\mathrm{t}=1 \mathrm{~s}$

Figure 10. Instantaneous deformation results for flexible membrane wing for $\mathrm{Re}=50000$ and $\alpha=16^{\circ}$ 
As seen from Figure 10 to Figure 12, deformation results for $\mathrm{Re}=50000$ with same angles of attack. The instantaneous displacements increase with increasing angles of attack as seen from Figure 10 to Figure 12. Shape of instantaneous deformation contour changed with formation of tip vortices. Deformations decreased towards to the trailing edge of membrane wing due to the effects of tip vortices.

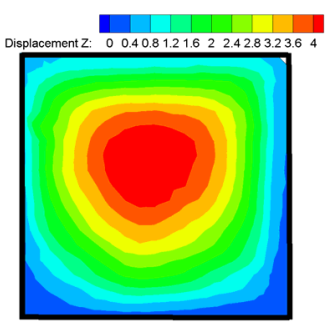

a) $t=0.001 s$

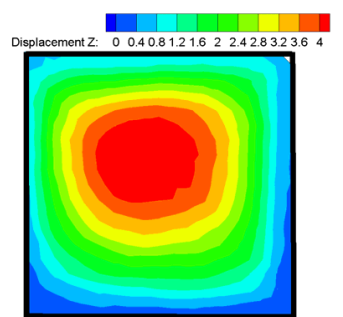

b) $\mathrm{t}=0.5 \mathrm{~s}$

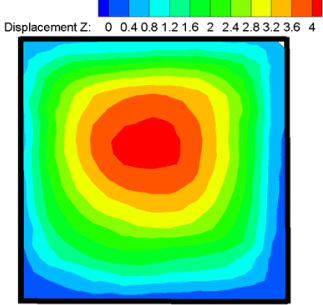

c) $t=1 \mathrm{~s}$

Figure 11. Instantaneous deformation results for flexible membrane wing for $\mathrm{Re}=50000$ and $\alpha=20^{\circ}$

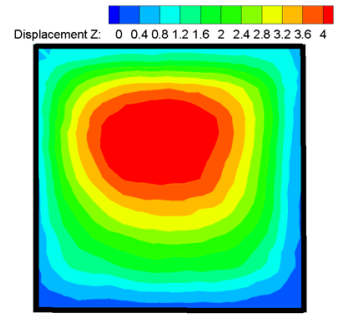

a) $\mathrm{t}=0.001 \mathrm{~s}$

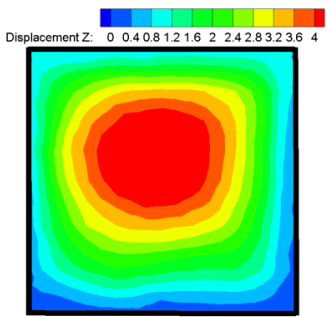

b) $\mathrm{t}=0.5 \mathrm{~s}$

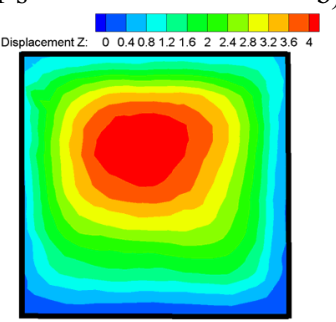

c) $\mathrm{t}=1 \mathrm{~s}$

Figure 12. Instantaneous deformation results for flexible membrane wing for $\mathrm{Re}=50000$ and $\alpha=24^{\circ}$

Figure 13 demonstrates the results of standard deviation of membrane wing in the direction normal to the chord-line. At angle of attack of $2^{\circ}$, mode of five was seen chordwise direction whereas second mode vibration was seen in spanwise especially at trailing edge of wing due to the effect of tip vortices. An increase in angle of attack caused to decrease in vibrational mode and the region of maximum standard deviation approached to the trailing edge.
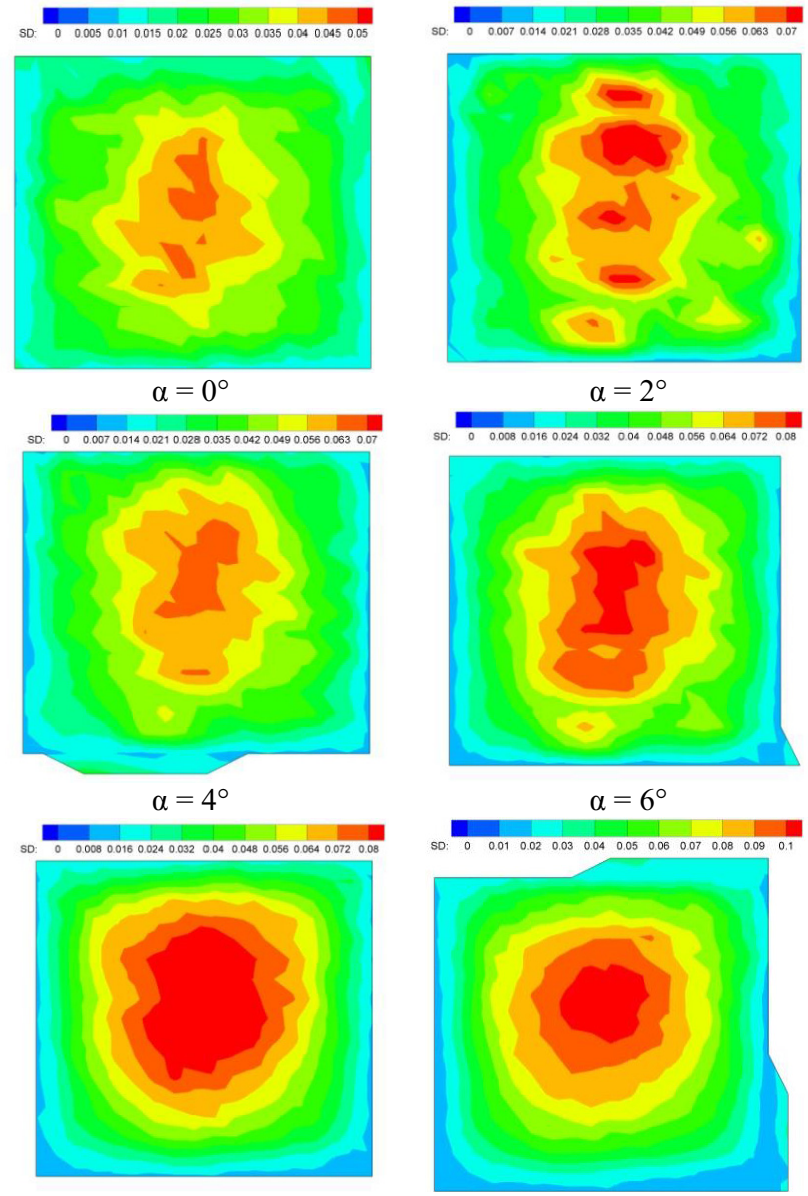

$\alpha=8^{\circ}$

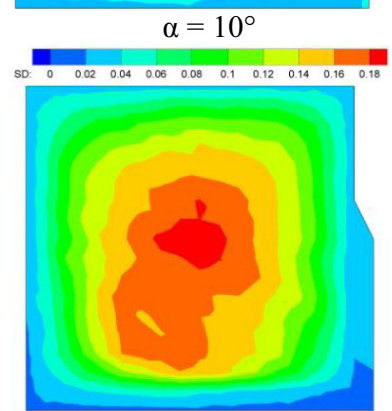

$\alpha=16^{\circ}$

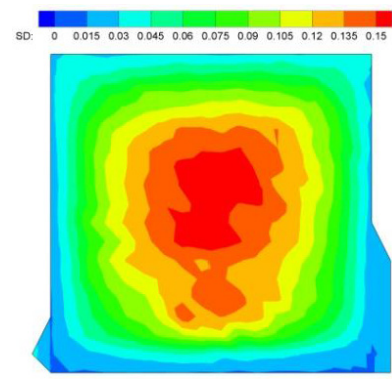

$\alpha=14^{\circ}$

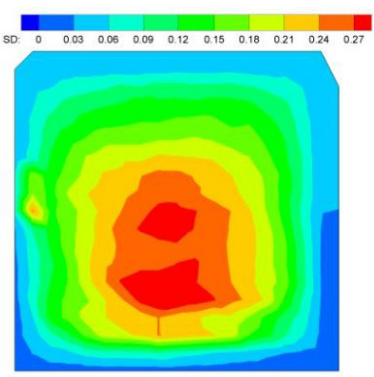

$\alpha=18^{\circ}$

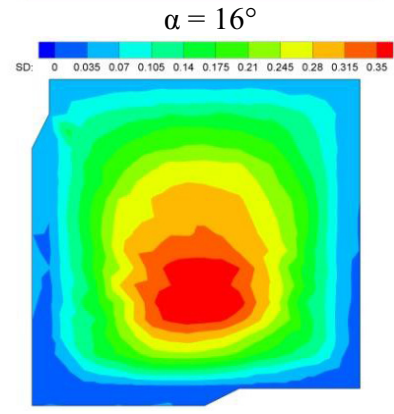

$\alpha=22^{\circ}$

Figure 13. Standard deviation measurement results of membrane wing for $\mathrm{AR}=1$ and $\mathrm{Re}=50000$.

\section{Conclusion}

Unsteady flow around flexible membrane wings at low Reynolds numbers of 25000 and 50000 was investigated experimentally. Smoke-wire technique for flow visualization was utilized. Instantaneous deformation measurements of membrane wing which were performed 
by means of DIC system were combined with the flow field measurements. It was deduced that increasing angle of attack caused to increase in membrane deformation. Analysis of the instantaneous deformation revealed chordwise and spanwise, which were because of shedding of leading-edge vortices as well as tip vortices. Consequently, vibrational mode decreased and maximum standard deviation location approached to the trailing edge due to increasing angle of attack.

\section{Acknowledgments}

The authors would like to acknowledge funding from the Scientific and Technological Research Council of Turkey (TÜBITAK) under the project no: 213M329, the Scientific Research Projects Unit of Erciyes University under the contract no: FDK-2015-6171, FBG-2014-5337 and FDA-2014-5273.

\section{References}

1. M.S. Genc, U. Kaynak, G.D. Lock, Flow over an Aerofoil without and with Leading Edge Slat at a Transitional Reynolds Number. Proc IMechE, Part G: J Aerospace Eng. 223(3), 217-231 (2009)

2. M.S. Genc, I. Karasu, H.H. Acıkel, M.T. Akpolat, Low Reynolds number flows and transition, in: M. Serdar Genc (Ed.), Low Reynolds Number Aerodynamics and Transition, Intech-Sciyo Publishing, ISBN 979-953-307627-9 (2012)

3. M.S. Genc, U. Kaynak, H. Yapıcı, Performance of transition model for predicting low $\mathrm{Re}$ aerofoil flows without/with single and simultaneous blowing and suction. Eur J Mech B-Fluid. 30(2), 218-235 (2011)

4. M.S. Genc, I. Karasu, H.H. Acıkel, An experimental study on aerodynamics of NACA2415 aerofoil at low Re numbers. Exp Therm Fluid Sci. 39, 252-264 (2012)

5. I. Karasu, M. S. Genç, H. H. Açikel, Numerical study on low Reynolds number flows over an Aerofoil. J. Appl. Mech. Eng. 2, 131 (2013)

6. W. Shyy, M. Berg, D. Ljungqvist, Flapping and flexible wings for biological and micro air vehicles. Prog Aerosp Sci. 35, 455-505 (1999)

7. M.S. Genç, Unsteady aerodynamics and flow-induced vibrations of a low aspect ratio rectangular membrane wing with excess length. Exp. Therm Fluid Sci. 44, 749759 (2013)

8. P. Rojratsirikul, Z. Wang, I. Gursul, Effect of prestrain and excess length on unsteady fluid-structure interactions of membrane airfoils. J Fluid Struct. 26(3), 359-376 (2010)

9. P. Rojratsirikul, M.S. Genc, Z. Wang, I. Gursul, Flowinduced vibrations of low aspect ratio rectangular membrane wings. J Fluid Struct. 27, 1296-1309 (2011)

10. B. Béguin, C. Breitsamter, Effects of membrane prestress on the aerodynamic characteristics of an elastoflexible morphing wing. Aerosp. Sci. Technol. 37, 138150 (2014)

11.S. Arbós-Torrent, B. Ganapathisubramani, R. Palacios, Leading-and trailing-edge effects on the aeromechanics of membrane aerofoils. J Fluid Struct. 38, 107-126 (2013)

12. R. Albertani, B. Stanford, J.P. Hubner, P.G. Ifju, Aerodynamic coefficients and deformation measurements on flexible micro air vehicle wings. Exp. Mech. 47(5), 625-635 (2007)

13. Y. Lian, W. Shyy, D. Viieru, B. Zhang, Membrane wing aerodynamics for micro air vehicles. Prog. Aerosp. Sci. 39(6), 425-465 (2003)

14. H. Aono, S.K. Chimakurthi, C.E. Cesnik, H. Liu, W. Shyy, Computational modeling of spanwise flexibility effects on flapping wing aerodynamics. In 47th AIAA aerospace sciences meeting including the new horizons forum and aerospace exposition, 18 (2009)

15. C.H. Kuo, J.K. Hsieh, Unsteady flow structure and vorticity convection over the airfoil oscillating at high reduced frequency. Exp. Therm Fluid Sci. 24(3), 117-129 (2001)

16. M.J. Ringuette, M. Milano, M. Gharib, Role of the tip vortex in the force generation of low-aspect-ratio normal flat plates. J. Fluid Mech. 581, 453-468 (2007) 